\title{
Validación del uso de teléfonos inteligentes para medición de ruido ambiental urbano
}

\section{Validation of the use of smartphones for measuring environmental urban noise}

\author{
Andrea Sangurima ${ }^{1}$, Michelle Medina ${ }^{1}$, María-Laura Guerrero ${ }^{2}$ (D), Daniel Orellana ${ }^{2,3}$ (D) \\ ${ }^{1}$ Carrera de Ingeniería Ambiental, Facultad de Ciencias Químicas, Universidad de Cuenca, Ecuador \\ ${ }^{2}$ LlactaLAB-Ciudades Sustentables, Departamento Interdisciplinario de Espacio y Población, Universidad de Cuenca, \\ Ecuador. \\ ${ }^{3}$ Facultad de Ciencias Agropecuarias, Universidad de Cuenca, Ecuador. \\ Autor de correspondencia: daniel.orellana@ucuenca.edu.ec \\ Fecha de recepción: 2 de octubre de 2020 - Fecha de aceptación: 15 de noviembre de 2020
}

\section{RESUMEN}

El presente estudio evalúa la fiabilidad del uso de teléfonos inteligentes para monitorear el ruido urbano, como una alternativa de bajo costo a los instrumentos de monitoreo tradicionales. La metodología consistió en diseñar una herramienta de evaluación de ruido en KoBoToolbox, la cual registró las mediciones recopiladas simultáneamente con un sonómetro y dos aplicaciones para teléfonos inteligentes, Sound Meter X Standard y Sound Meter Pro, en los sistemas iOS y Android respectivamente. Las tres mediciones fueron comparadas con el método de análisis de varianza (ANOVA) de un factor. Las pruebas se realizaron en el parque El Paraíso, de la ciudad de Cuenca, basándose en la legislación ecuatoriana. Los resultados sugieren que la aplicación utilizada para la plataforma Android presenta menor grado de variación de medición con respecto a la utilizada en la plataforma iOS. Así también, muestran una relación significativa entre niveles de ruido elevados y focos de contaminación acústica por presencia de tráfico vehicular. La investigación tiene implicaciones para el uso futuro en programas de monitoreo participativo de ruido ambiente bajo un enfoque de ciencia ciudadana, complementando las normas y legislaciones vigentes. Palabras clave: Aplicación, teléfono inteligente, ruido urbano, KoBoToolbox, espacio público.

\begin{abstract}
This study evaluates the reliability of using smartphones for monitoring environmental urban noise as a low-cost alternative to conventional monitoring instruments. A noise evaluation tool was designed using KoBoToolbox enabling the simultaneous recording of sound measurements with a professional sound level meter and two smartphone apps: Sound Meter X Standard on iOS, and Sound Meter Pro on Android. The measurements were compared with the one-factor analysis of variance (ANOVA) method. Sampling was carried out in "El Paraíso" park in Cuenca, by following the Ecuadorian legislation. Results suggest that Android app shows less measurement variation compared to the iOS platform. Results also showed a significant relation between high noise levels and noise pollution hotspots caused by traffic. Indeed, this research establishes a background for future use in noise level community monitoring programs under a citizen science focus, while also complements current national laws and regulations.
\end{abstract}

Keywords: App, smartphone, urban noise, KoBoToolbox, public space.

\section{INTRODUCCIÓN}

La contaminación acústica en el casco urbano de las ciudades es un tema de gran impacto e importancia puesto que, el ruido ambiental es uno de los principales problemas de contaminación ambiental urbana ya que interfiere en las actividades de la población, perturbando su bienestar en general. Su fuente primaria radica en el parque automotor (Bocher, Petit, Picaut, Fortin, \& Guillaume, 2017). Debido a esto, varios países han adoptado medidas para cuantificar y controlar el nivel de ruido a través de regulaciones y del establecimiento de métodos de medición estandarizados, los cuales dependen de instrumentos especializados como sonómetros y dosímetros, que son de alto costo, difícil acceso y manejo, por lo que su uso está restringido a entidades especializadas (Wessels \& Basten, 2016).

Por ese motivo, la medición de ruido ambiente suele depender de campañas y programas específicos, diseñados para tener datos representativos a nivel de ciudad (Motta, 2020). Sin embargo, existe una gran cantidad de casos en los que se requiere mediciones puntuales de ruido ambiente para contrastar con los valores límites permisibles, tales como la evaluación de parques y 
espacios públicos, campus universitarios, centros de ocio y comercio, y otros lugares colectivos. En estos casos, la disponibilidad de datos de ruido ambiente y la posibilidad de levantar mediciones estandarizadas suele ser muy baja o nula; y en los pocos programas de monitoreo que se han realizado a lo largo del tiempo, se ha descubierto que el $97 \%$ de la población en las urbes experimentan exposiciones a niveles de ruido mayores a $55 \mathrm{~dB}^{1}(\mathrm{~A})^{2}$ (GAD Municipal del Cantón Cuenca, 2016), que acorde con la Organización Mundial de la Salud (OMS), es nocivo. Y conforme a Trombetta Zannin, Coelho Ferreira, \& Szeremetta (2006) los parques urbanos, pese a que suponen un papel esencial en el desarrollo de localidad, figuran como focos de contaminación acústica (Maristany, 2016).

La importancia de establecer estas bases de datos certeros radica en las alternativas de uso que presentan como: la determinación de los puntos críticos para adoptar las medidas de mitigación pertinentes (Maheswaran, Nishant, Senthil Murugan, \& Prabaharan, 2020), para apoyar el control de los planes ya convenidos y aplicados (Džambas \& Dragčević 2020), y para elaboración de modelos predictivos (Coral, Moromenacho, Moreta, Villalba, \& Oviedo, 2020). Por ese motivo, medidas convenientes y de bajo costo que ayuden a obtener esta información son de gran interés para personas particulares, familias, asociaciones barriales u organizaciones gubernamentales, entre otras.

Los avances tecnológicos en el desarrollo de dispositivos móviles, en particular de teléfonos inteligentes y aplicaciones de uso práctico han permitido que el uso de los mismos no se limite únicamente a la comunicación. La portabilidad y sinergia con los sensores que poseen, como micrófono, cámara, sistema de posicionamiento global (GPS), acelerómetros, entre otros (Garg, Lim, \& Lee, 2019), los convierten en instrumentos útiles para realizar monitoreos ambientales (Zuo, Xia, Liu, \& Qiao, 2016). Estudios previos, han demostrado que es posible medir niveles de ruido ambiental mediante aplicaciones de dispositivos móviles con una precisión equiparable a los instrumentos profesionales (Aumond et al., 2017; Lefevre \& Issarny, 2018; McLennon, Patel, Behar, \& AbdoliEramaki, 2019; Murphy \& King, 2016b). Existen variedades de aplicaciones de medición de sonido tanto para el sistema operativo Android como para iOS, pero solo una fracción logran la precisión suficiente para evaluar los niveles de ruido (Murphy \& King, 2016b).

En Ecuador, el uso de dispositivos móviles inteligentes se ha elevado significativamente en los últimos años. Según la Encuesta Nacional de Empleo, Desempleo y Subempleo (ENEMDU) realizada por el Instituto Nacional de Estadística y Censos (INEC), del 2011 al 2013 el porcentaje de personas con teléfonos inteligentes no era mayor al $16.9 \%$, mientras que para el 2018 el $70.2 \%$ de la población poseía un teléfono inteligente (Instituto Nacional de Estadística y Censos, 2018; Ministerio de Telecomunicaciones y Sociedad de la Información, 2018), por lo que la factibilidad de monitoreo ciudadano de la contaminación acústica puede ser muy alta. Los sistemas operativos móviles más comunes en el mercado son Android y iOS (Celestina, Hrovat, \& Kardous, 2018), e investigaciones preliminares han encontrado que la

\footnotetext{
${ }^{1} \mathrm{~dB}$ (decibelio) es una unidad utilizada para medir generalmente la intesidad del sonido.

${ }^{2} \mathrm{~dB}(\mathrm{~A})$ es una unidad adimensional que expresa el logaritmo de la razón entre una cantidad medida y una cantidad de
}

medición de ruido con sistemas basados en iOS produce menores diferencias con respecto a valores de referencia (Kardous \& Shaw, 2014).

Al considerar diversos estudios donde se demuestra la viabilidad del uso aplicaciones y programas en teléfonos inteligentes para obtener mapas y mediciones de ruido certeros (Eißfeldt, 2020; Garg et al., 2019; Kanjo, 2010; Lee, Garg, \& Lim, 2020; Murphy \& King, 2016a); y que al integrarlo con KoBoToolbox dinamizaría el proceso de recolección de información de datos masiva para este tipo de procedimientos, podemos explorar la posibilidad de usarlos para la recopilación de información acústica puntual en espacios urbanos por parte de ciudadanos, como complemento de las campañas y programas especializados de monitoreo de contaminación acústica. De esta manera sería posible para los usuarios adquirir información sobre la calidad acústica del entorno de forma puntual y crear conciencia de la realidad en cuanto a temas ambientales para tomar las medidas pertinentes.

El objetivo de este estudio comparativo es constatar la viabilidad y precisión del uso de dos aplicaciones de teléfonos inteligentes, en conjunto con la plataforma KoBoToolbox para sondeos de ruido ambiental en espacios públicos, como alternativa a los instrumentos de medición convencionales. Para esto, se analizan las diferencias estadísticas en cuanto a la exactitud de medición para las aplicaciones móviles Sound Meter X Standard y Sound Meter Pro, en sistemas iOS y Android respectivamente, con respecto a las mediciones realizadas con un sonómetro profesional, siguiendo el procedimiento estándar del país. Esto permitirá implementar programas de monitoreo participativo de ruido ambiente bajo un enfoque de ciencia ciudadana, con una herramienta adaptable a las diversas metodologías empleadas para este fin, como complemento a normas y legislaciones vigentes nacionales referentes al ruido ambiente.

\section{MÉTODOS}

Para establecer la viabilidad de dispositivos móviles como medidores de contaminación acústica, se compararon las mediciones simultáneas de ruido ambiente en espacios públicos obtenidas con Sound Meter X Standard para iOS y Sound Meter Pro para Android con mediciones de referencia obtenidas mediante un sonómetro integrador de clase 2, marca CENTER TECHNOLOGY CORP, modelo 390. Dicho artefacto cumplía con los requisitos de la Norma de la Comisión Electrotécnica Internacional IEC 61672-1:2013 (International Electrotechnical Commission (IEC), 2013) y ANSI/ASA S1.4-2014 (Acoustical Society of America (ASA, 2014)).

La calibración es un factor importante que influye significativamente en un registro adecuado de los niveles de presión sonora y el espectro de frecuencia (Garg et al., 2019). En consecuencia, fue necesario calibrar los micrófonos de los teléfonos inteligentes previo a las mediciones de campo; con la suscripción a Sound Meter Pro para iOS se produjo un tono de referencia de $1 \mathrm{kHz}$ para la calibración del micrófono integrado, esta señal fue

referencia para describir los niveles de presión sonora, corregido para adaptar estos valores a la sensibilidad del oído humano 
conectada a un altavoz y posterior se colocó el micrófono de los dispositivos lo más cerca posible de la fuente, con una posición y orientación adecuada (Serpanos, Renne, Schoepflin, \& Davis, 2018).

A continuación, a través de la opción de configuración de las aplicaciones seleccionamos la opción de calibración en donde se pudo visualizar el nivel de sonido de entrada medido como un valor de texto, de este modo se consideró que si el nivel de entrada medido concordaba con el nivel de referencia emitido entonces el micrófono se encontraba calibrado, caso contrario se ajustó manualmente el nivel de entrada con el nivel de referencia (Faber Acoustical, LLC, 2019). En caso de no contar con la suscripción mencionada anteriormente, se recomienda utilizar el programa de uso libre Audacity para generar el tono de referencia requerido. Para una mejor ejecución de la calibración, es preferible realizar este procedimiento en una habitación o lugar tranquilo, con las mínimas influencias de sonidos externos (Crook, 2020; Rana, Chou, Bulusu, Kanhere, \& Hu, 2015). En la Tabla 1 se presenta información detallada de los equipos utilizados.

Para la recolección de datos en campo, primero se configuró KoBoToolbox, una herramienta de código abierto que permite recolectar información rápidamente in situ y exportarla, permitiendo establecer una base de datos. En esta plataforma se diseñó la metodología a seguir y se fundamentó en la normativa ecuatoriana establecida en el Anexo 5 del Texto Unificado de Legislación Secundaria para determinar el nivel de ruido para fuentes fijas (Ministerio del Ambiente, 2015). Se esquematizó un formulario de evaluación que registró todos los datos para los 3 tipos de mediciones realizados, calculó automáticamente el ruido total utilizando la fórmula de cálculo de nivel presión sonora equivalente (LeqProm) y se ubicó en una escala predefinida el nivel de ruido encontrado.

$$
\text { LeqProm }=10 \log \left[\frac{1}{n_{i}} *\left(\begin{array}{c}
10^{0.1 \text { Leq }_{1}}+10^{0.1 \text { Leq }_{2}} \\
+\cdots+10^{0.1 \text { Leq }_{n}}
\end{array}\right)\right]
$$

Donde $n$, corresponde al número de mediciones, $L$ nivel de presión sonora, $e q$ se atribuye a equivalente $\mathrm{y}, p$ es el promedio de las muestras Leq (promedio logarítmico).

Las mediciones fueron realizadas simultáneamente con dos aplicaciones móviles, usando las versiones de pago de las mismas para facilitar el proceso de recolección de datos debido a que dichas versiones permitían preestablecer el tiempo de medición de cada muestra; y se instalaron en distintos dispositivos de diferente sistema operativo. Para su selección, se usó como base los resultados de estudios previos ya mencionados (Murphy \& King, 2016b; Nast, Speer, \& Prell, 2014), considerando su disponibilidad para nuestra región. Así, se seleccionaron Sound Meter X Standard para iOS y Sound Meter Pro para Android.

Se seleccionó el Parque El Paraíso, uno de los principales parques de la ciudad de Cuenca porque se encuentra en una zona donde la exposición al ruido oscila entre los 60 y $65 \mathrm{~dB}(\mathrm{~A})$ en el periodo diurno, según el informe de Monitoreo de Ruido Ambiente en la ciudad de Cuenca para el año 2018 (GAD Municipal del Cantón Cuenca \& Universidad del Azuay, 2018), en el que, el máximo permisible es $55 \mathrm{~dB}(\mathrm{~A})$ (Ministerio del Ambiente, 2015). Y la medición en campo se ejecutó durante los días 19 y 23 de septiembre de 2019, en el horario comprendido de $07 \mathrm{~h} 01$ a $21 \mathrm{~h} 00$.

La determinación de los puntos de medición se elaboró según el muestreo de conglomerados, para ello se consideró como factores la delimitación geográfica del área de interés y el número mínimo de puntos para representar adecuadamente la distribución de una variable (Lind, Mason, \& Marchal, 2004). Se definieron 30 puntos de medición posicionados en su mayoría a lo largo de caminos peatonales preferenciales dentro del parque, contemplando las zonas de mayor afluencia de usuarios y mayoritariamente adyacentes a calles con circulación vehicular.

Los niveles de ruido se determinaron acatando el procedimiento estipulado en la legislación ecuatoriana, midiendo el valor de Nivel de Presión Sonora Equivalente (Leq) para fuentes fijas. Con ello, se obtuvieron valores de 15 segundos cada una con una pausa de aproximadamente 10 segundos entre cada medición; para cada punto y horario determinado en base a las cada una. Los instrumentos empleados fueron colocados en ponderación (A), utilizada para control de ruido urbano por la semejanza al oído humano en la percepción de sonidos, y modo de respuesta lenta dado que esta modalidad arroja valores cada segundo. En la primera mitad del análisis, todos los equipos fueron soportados en trípodes, modelo Slik f740, a una altura de $1.5 \mathrm{~m}$ (Montes González, Barrigón Morillas, Rey Gozalo, \& Godinho, 2020) y distancia del equipo al momento de la medición de $1.0 \mathrm{~m}$, como se establece en el Anexo 5 del Texto Unificado de Legislación Secundaria para determinar el nivel de ruido para fuentes fijas citado previamente.

Tabla 1. Información de los teléfonos inteligentes y aplicaciones utilizadas.

\begin{tabular}{|c|c|c|c|c|c|c|}
\hline Equipo & & & & & Origen & Normativas \\
\hline Sonómetro & Center & anology & Cent & 390 & Taiwán & $\begin{array}{c}\text { IEC61672-1 Clase } 2 \\
\text { ANSI S1.4 Tipo } 2\end{array}$ \\
\hline Equipo & Marca & Modelo & Año & & Aplicacic & \\
\hline \multirow{8}{*}{$\begin{array}{l}\text { Teléfono } \\
\text { inteligente }\end{array}$} & \multirow{4}{*}{ iPhone } & \multirow{4}{*}{$6 s$} & \multirow{4}{*}{2015} & \multirow{4}{*}{$\begin{array}{r}\text { Sound Meter } \\
\text { X Standard }\end{array}$} & \multicolumn{2}{|c|}{ Características } \\
\hline & & & & & Desarrollador & Faber Acoustical \\
\hline & & & & & Versión & 10.5 .2 \\
\hline & & & & & Costo & $\$ 0.99 / \mathrm{mes}$ \\
\hline & \multirow{4}{*}{ Huawei } & \multirow{4}{*}{$\begin{array}{c}\text { Mate } 10 \\
\text { Pro }\end{array}$} & \multirow{4}{*}{2017} & \multirow{4}{*}{$\begin{array}{r}\text { Sound Meter } \\
\text { Pro }\end{array}$} & \multicolumn{2}{|c|}{ Características } \\
\hline & & & & & Desarrollador & Smart Tools Co. \\
\hline & & & & & Versión & 2.5 .10 \\
\hline & & & & & Costo & $\$ 1.56$ \\
\hline
\end{tabular}


Tabla 2. Análisis ANOVA por diferentes equipos de medición de ruido.

\begin{tabular}{cccccc}
\hline Fuente de variación & $\begin{array}{c}\text { Suma de los } \\
\text { cuadrados }\end{array}$ & Grados de libertad & Cuadrados medíos & F & Valor $p$ \\
\hline Total & $2,408.10$ & 89 & 27.06 & - & - \\
Tratamiento & 16.80 & 2 & 8.40 & 0.09 & 0.7375 \\
Error & $2,391.30$ & 87 & - & - & - \\
\hline
\end{tabular}

Tabla 3. Análisis descriptivo de la diferencia media de medición de ruido.

\begin{tabular}{cccccc}
\hline $\begin{array}{c}\text { Valor de referencia } \\
\mathrm{dB}(\mathrm{A})\end{array}$ & $\mathrm{N}$ & $\begin{array}{c}\text { Diferencia media con respecto al } \\
\text { valor referencial }\end{array}$ & $\begin{array}{c}\text { Desviación } \\
\text { estándar }\end{array}$ & $\begin{array}{c}\text { Error estándar de la } \\
\text { media }\end{array}$ & Rango \\
\hline $50-55$ & 12 & 2.5 & 1.95 & 0.56 & 12 \\
$56-60$ & 8 & 2 & 2.47 & 0.87 & 10 \\
$>60$ & 10 & 4.3 & 4.56 & 1.44 & 17 \\
\hline
\end{tabular}

Mientras que, en la segunda mitad, únicamente el sonómetro integrador continuó soportado; debido a que en gran parte de los proyectos de ciencia ciudadana relacionados a este campo sujetan el dispositivo móvil con la mano, tomando en cuenta siempre ciertas condiciones para obtener una medición real de ruido in-situ (Picaut $e t$ al., 2019). Luego, en KoBoToolbox se registraron los valores de niveles de ruido marcados en los dispositivos de cada punto crítico, y esta plataforma calculó automáticamente el promedio niveles de ruido marcados en los dispositivos de cada punto crítico, y esta plataforma calculó automáticamente el promedio logarítmico (LeqProm) de los datos obtenidos, mediante la ecuación referida en el Anexo 5 del TULSMA del Ministerio del Ambiente (2015).

Para evaluar la existencia de diferencias estadísticas significativas en la precisión de medición de los 240 datos levantados durante el horario de muestro establecido, por cada punto crítico del Parque El Paraíso, se trabajó con un Análisis de Varianza (ANOVA) de un factor, que en este caso fue el equipo de medición de ruido, el cual poseía tres variantes: dos dispositivos móviles y el sonómetro. De la misma manera, para determinar el rendimiento de las aplicaciones móviles y conocer la magnitud del error de medición con respecto al sonómetro, se realizó un análisis de estadística descriptiva.

Por otro lado, se trabajó con diagramas de caja para observar a grosso modo la distribución de los datos y así conocer la influencia del tráfico vehicular en la generación de niveles de ruido elevados, así se categorizó los puntos críticos distinguiendo su cercanía a calles transitadas. Todos los análisis se realizaron con el programa RStudio versión 1.2.1335.

\section{RESULTADOS}

En la Tabla 2 se muestra que el valor calculado de $\mathrm{F}$ fue 0.09 resultando menor al valor crítico de 3.95 , además el valor de $p$ fue mayor que el nivel de significancia de 0.05 $(\alpha=0.05)$, por lo que las diferencias entre las medias no fueron estadísticamente significativas de modo que se puede afirmar que no existe diferencia significativa entre las mediciones de ruido realizadas por los tres distintos equipos.

En la Tabla 3 se expresa mediante un análisis descriptivo la diferencia media entre los valores medidos con los teléfonos inteligentes y el valor de referencia considerado, en este caso como el sonómetro. Se puede observar que entre los 50 y $56 \mathrm{~dB}(\mathrm{~A})$ la diferencia media en la medición de las aplicaciones con respecto a las condiciones de referencia es de 2.5 y 2 respectivamente, mientras que para mediciones mayores a $60 \mathrm{~dB}(\mathrm{~A})$ el resultado de la media es más variable.

Del tamaño de muestra $(n=30)$, se determinaron 18 puntos lejanos de calles transitadas y 12 puntos cercanos a las mismas. Como resultado de la interpretación de los datos registrados alejados de focos de tráfico vehicular comparando las mediciones registradas por el Sonómetro, Android y iOS se observó en la Figura 1 que los niveles de ruido oscilan en un rango de $52 \mathrm{a} 56 \mathrm{~dB}(\mathrm{~A})$ con valores máximos de $61 \mathrm{~dB}(\mathrm{~A})$ aproximadamente. Por otra parte, en la interpretación de los datos registrados cercanos a focos de tráfico vehicular se observó en la Figura 1 que los niveles de ruido registrados se situaron en un rango 57 a $67 \mathrm{~dB}(\mathrm{~A})$ con valores máximos de $74 \mathrm{~dB}(\mathrm{~A})$ aproximadamente.

Adicionalmente se observa por un lado que, existe una tendencia de los datos a una distribución positivamente asimétrica, ya que existió una cercanía de la mediana al primer cuartil. Por otra parte, se muestra que la aplicación con menor grado de variabilidad está asociada a la plataforma Android, ya que la asociada a la plataforma iOS presenta una distribución de datos más variada con respecto al sonómetro (Fig. 1).

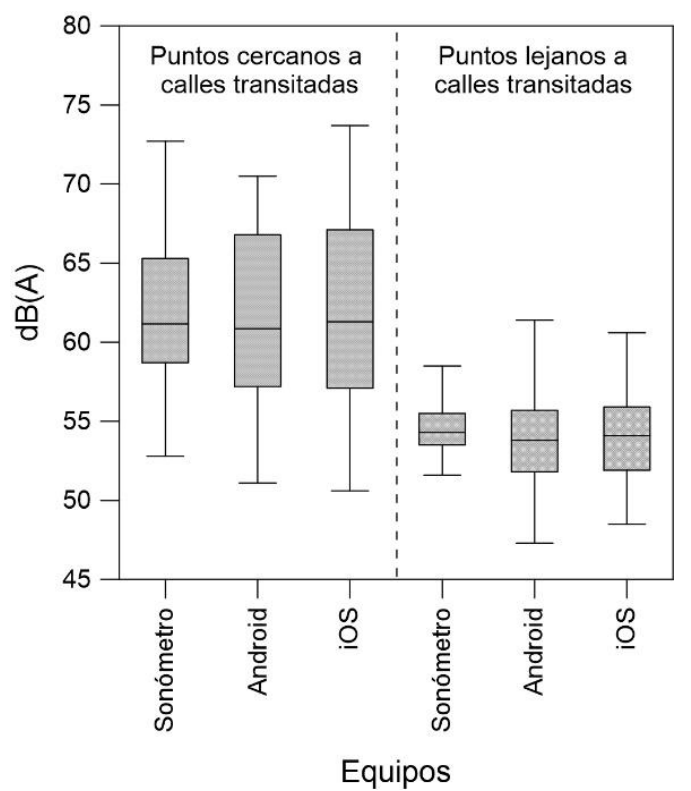

Figura 1. Diagrama de caja de los niveles de ruido registrados lejanos y cercanos a calles transitadas. 


\section{DISCUSIÓN}

El estudio demuestra que, para la medición de niveles de ruido ambiente en espacios públicos con fines informativos, es posible reemplazar el uso del sonómetro por teléfonos inteligentes a través de las aplicaciones Sound Meter X Standard y Sound Meter Pro, con la ayuda de KoBoToolbox. De este modo, se facilita a la población la accesibilidad a herramientas de determinación de niveles de ruido urbano, ya que no depende de la adquisición de un equipo profesional para realizar las mediciones.

Además, con la determinación del nivel de significancia estadística, se confirma la confiabilidad de medición de los dispositivos móviles, cuyo uso está sujeto a una previa capacitación para la aplicación correcta de la metodología. Estos hallazgos apoyan estudios que proponen la adopción generalizada de teléfonos inteligentes como dispositivos de detección colectivos (D'Hondt, Stevens, \& Jacobs, 2013; Eißfeldt, 2020; McLennon et al., 2019; Zamora, Calafate, Cano, \& Manzoni, 2017) y promueven la participación de la sociedad en el control de la contaminación acústica, en espacios abiertos (Aumond $e t$ al., 2017).

Adicionalmente, se determina una distribución de datos de mayor grado de variabilidad en la plataforma iOS con respecto al sonómetro, por lo que se recomienda usar Android. De igual manera, las diferencias medias de medición encontradas denotan una semejanza entre los datos registrados por los distintos equipos; aun así, comparando la variabilidad de error estándar se muestra que las aplicaciones son menos eficientes midiendo niveles de ruido elevado. A pesar de esto las aplicaciones realizan un trabajo adecuado al medir dentro de un grado de error aceptable que es típicamente de $\pm 2 \mathrm{~dB}(\mathrm{~A})$ (Murphy \& King, 2016b). Con ello podemos mencionar que, en condiciones reales la precisión de medición variará según el dispositivo que se esté empleando, influida por la calidad del micrófono (Picaut et al., 2019).

De igual manera, se pudo ratificar la eficacia de las mediciones con valores certeros. Complementado el análisis de Murphy \& King (2016b), así como el de McLennon et al. (2019) con respecto al hecho de que las aplicaciones no están listas para reemplazar a los equipos de medición convencionales.

Por otro lado, mediante diagramas de caja se comprueba una relación entre niveles de ruido elevados y focos de contaminación acústica por presencia de tráfico vehicular en la urbe, corroborando el hecho de que el ruido urbano es proveniente en su mayoría del parque automotor (Maristany, 2016). Ya que, los valores de ruido encontrados, en su mayoría han superado el nivel permisible para esa zona de $55 \mathrm{~dB}(\mathrm{~A})$; implicando una afectación a la salud física (enfermedades cardiovasculares) y psicosocial (trastornos de sueño) al estar expuestos a esos niveles manera continua (Johansson, 2020; Zamorano Gonzáleg et al., 2019). Demostrando que existe una relación directa con la calidad de vida de los ciudadanos, de igual manera limita el uso del espacio por lo poco satisfactorio y a largo plazo peligroso, que resulta.

Cabe destacar que en el estudio solamente se midió el ruido total. En cuanto al ruido de fondo proveniente de las conversaciones, no se consideró para el estudio, dado que no fue posible determinarlo porque en la normativa ecuatoriana, no está claro el procedimiento a seguir. Por último, antes de desarrollar un estudio encaminado a este tema, es aconsejable efectuar un recorrido previo al sito, para determinar las zonas de posible interferencia con el instrumento de medición, como los postes de alta tensión eléctrica. Estos afectan principalmente a sonómetros y dificultan la toma de medición.

\section{CONCLUSIONES}

Es innegable que, en la ciudad de Cuenca el uso de mapas de ruido para calificar el grado de polución nos proporciona una idea general de calidad del entorno sonoro. Pero el uso de otras herramientas y metodologías, como la propuesta en esta investigación, nos ha permitido procesar con mayor agilidad temas de esta índole, además de establecer valores más puntuales; en especial en espacios públicos donde la recolección de esta información es complicada y poco precisa.

Debido a el potencial que han mostrado estas aplicaciones: Sound Meter X Standard y Sound Meter Pro, con la ayuda de KoBoToolbox para ser utilizada como instrumento de medición popular, se ha probado que es posible tener resultados verídicos siguiendo procedimientos estandarizados con los dispositivos móviles, sin llegar a ser complicada su ejecución o requerir equipos de alto costo.

Con ello, nos permitiría la creación de una planificación urbana sostenible a nivel sectorial o barrial, así como de políticas públicas, centradas en minimizar la exposición de los espacios públicos al ruido de carretera; la cual podría contener propuestas de control, preventivas y correctivas; como la integración de vegetación cuya función sería de amortiguar los sonidos desagradables y mejorar el confort urbano/paisajístico.

Finalmente, concluimos que la principal limitación de este estudio fue la falta de profundidad en las especificaciones de los dispositivos móviles en los resultados. Como sugerencia para futuras investigaciones se considera que es importante reunir varios dispositivos móviles de diferentes fabricantes, y asociar sus características con los datos recolectados.

\section{AGRADECIMIENTO}

Este trabajo forma parte del proyecto "Evaluación del Espacio Público Abierto (EPA) en la ciudad de Cuenca" y fue patrocinado por el Grupo Llactalab-Ciudades Sustentables del Departamento de Espacio y Población de la Universidad de Cuenca. Se remarca la contribución de Diana Moscoso, PhD docente de la Universidad de Cuenca, por sus valiosos consejos en cuanto a Calidad de Aire Ambiente; de igual manera al Ingeniero Javier Urgilés y René Fernández por su apoyo durante las excursiones para toma de mediciones. 


\section{REFERENCIAS}

Acoustical Society of America (ASA). (2014). American National Standard Electroacoustics - Sound Level Meters. Disponible en https://webstore.ansi.org/ preview-pages/ASA/preview_ANSI+ASA+S1.42014+Part+1+IEC+61672-1-2013+(R2019).pdf

Aumond, P., Lavandier, C., Ribeiro, C., Gonzalez Boix, E., Kambona, K., D’Hondt, E., \& Delaitre, P. (2017). A study of the accuracy of mobile technology for measuring urban noise pollution in large scale participatory sensing campaigns. Applied Acoustics, 117, 219-226. https://doi.org/10.1016/ j.apacoust.2016.07.011

Bocher, E., Petit, G., Picaut, J., Fortin, N., \& Guillaume, G. (2017). Collaborative noise data collected from smartphones. Data in Brief, 14, 498-503. https://doi.org/10.1016/j.dib.2017.07.039

Celestina, M., Hrovat, J., \& Kardous, C. A. (2018). Smartphone-based sound level measurement apps: Evaluation of compliance with international sound level meter standards. Applied Acoustics, 139, 119-28. https://doi.org/10.1016/j.apacoust.2018.04.011

Coral, K., Moromenacho, T., Moreta, A., Villalba, F. \& Oviedo, E. O. (2020). Modelos estadísticos de ruido ambiental para el Distrito Metropolitano de Quito DMQ, mediante datos históricos del 2009 al 2015, validados al 2019, como herramienta de calidad ambiental. ACI Avances En Ciencias E Ingenierías, 12(1), 24. https://doi.org/10.18272/aci.v12i1.941

Crook, J. (2020, 24 octubre). Home. Audacity ®. https://www.audacityteam.org/

D’Hondt, E., Stevens, M., \& Jacobs, A. (2013). Participatory noise mapping works! An evaluation of participatory sensing as an alternative to standard techniques for environmental monitoring. Pervasive and Mobile Computing, 9(5), 681-694. https://doi.org/10.1016/j.pmcj.2012.09.002

Džambas, T., \& Dragčević, V. (2020). Ocjena učinkovitosti mjera za smanjenje razina buke u urbanim sredinama. e-Zbornik: Elektronički Zbornik Radova Građevinskog Fakulteta, 10(19), 1-9.

Eißfeldt, H. (2020). Sustainable urban air mobility supported with participatory noise sensing. Sustainability, 12(8), 3320. https://doi.org/10.3390/su12083320

GAD Municipal del Cantón Cuenca (Ed.). (2016). Plan de desarrollo y ordenamiento territorial del cantón Cuenca 2015. Cuenca, Ecuador: GAD Municipal del Cantón Cuenca.

GAD Municipal del Cantón Cuenca, \& Universidad del Azuay. (2018). Ruido en Cuenca 2012-2018. Vol. 3. Cuenca, Ecuador: Universidad del Azuay Casa Editora.

Garg, S., Lim, K. M., \& Lee, H. P. (2019). An averaging method for accurately calibrating smartphone microphones for environmental noise measurement. Apple Acoustics, 143, 222-228. https://doi.org/10.1016/j.apacoust.2018.08.013

Instituto Nacional de Estadística y Censos (INEC). (2018). Tecnologías de la Información y Comunicación-TIC. Vol. 5.

International Electrotechnical Commission (IEC). (2013). Electroacoustics sound level meters. IEC
61672-1. Geneva, Switzerland: International Electrotechnical Commission.

Johansson, M. (2020). An indicative case-control study of noise perception due to environmental noise sources. Master thesis. KTH, School of Engineering Sciences (SCI), Engineering Mechanics, Fluid Mechanics and Engineering Acoustics, Marcus Wallenberg Laboratory, Gothenburg, Sweden.

Kanjo, E. (2010). NoiseSPY: A real-time mobile phone platform for urban noise monitoring and mapping. Mobile Networks and Applications, 15, 562-574. https://doi.org/10.1007/s11036-009-0217-y

Kardous, C. A., \& Shaw, P. B. (2014). Evaluation of smartphone sound measurement applications. The Journal of the Acoustical Society of America, 135(4), 186-192. https://doi.org/10.1121/1.4865269

Lee, H. P., Garg, S., \& Lim, K. M. (2020). Crowdsourcing of environmental noise map using calibrated smartphones. Applied Acoustics, 160, $107-$ 130. https://doi.org/10.1016/j.apacoust.2019.107130

Lefevre, B., \& Issarny, V. (2018). Matching technological societal innovations: The social design of a mobile collaborative app for urban noise monitoring. 2018 IEEE International Conference on Smart Computing (SMARTCOMP), Taormina, Italy. Pp.33-40.

Lind, D. A., Mason, R. D., \& Marchal, G. W. (2004). Estadística para administración y economía. Décima primera edición. México D. F., México: Alfaomega.

Maheswaran, K., Nishant, G. S., Senthil Murugan, S., \& Prabaharan, T. (2020). Acoustic level measurement by noise mapping method and mitigation using acoustic plaster in Power Press Industry. International Journal of Advanced Science and Technology, 29(7), 11200207.

Maristany, A. R. (2016). Paisaje sonoro urbano "Soundwalk" como método de análisis integral PENSUM, 2(2).

McLennon, T., Patel, S., Behar, A., \& Abdoli-Eramaki, M. (2019). Evaluation of smartphone sound level meter applications as a reliable tool for noise monitoring. Journal of Occupational and Environmental Hygiene, 16(9), 620-627. https://doi.org/10.1080/ 15459624.2019 .1639718

Ministerio de Telecomunicaciones y Sociedad de la Información. (2018). Libro Blanco de la Sociedad de la Información y del Conocimiento. Quito, Ecuador: Ministerio de Telecomunicaciones y Sociedad de la Información (MINTEL).

Ministerio del Ambiente. (2015). Texto Unificado de Legislación Secundaria del Ministerio del Ambiente (TULSMA), Libro VI, Anexo 5. № 387. Pp. 187.

Montes González, D., Barrigón Morillas, J. M., Rey Gozalo, G., \& Godinho, L. (2020). Evaluation of exposure to road traffic noise: Effects of microphone height and urban configuration. Environmental Research, 191, 110055. https://doi.org/10.1016/ j.envres.2020.110055

Motta, H. G. (2020). Comparación de los niveles de ruido, normativa y gestión de ruido ambiental en Lima y Callao respecto a otras ciudades de Latinoamérica. Revista Kawsaypacha: Sociedad y Medio Ambiente, 5, 107-142. 
Murphy, E., \& King, E. A. (2016a). Smartphone-based noise mapping: Integrating sound level meter app data into the strategic noise mapping process. The Science of the Total Environment, 562, 852-859. https://doi.org/10.1016/j.scitotenv.2016.04.076

Murphy, E., \& King, E. A. (2016b). Testing the accuracy of smartphones and sound level meter applications for measuring environmental noise. Applied Acoustics, $106,16-22$ https://doi.org/10.1016/j.apacoust.2015.12.012

Nast, D. R., Speer, W. S., \& Le Prell, C. G. (2014). Sound level measurements using smartphone "Apps": Useful or inaccurate? Noise and Health, 16(72), 251. https://doi.org/10.4103/1463-1741.140495

Picaut, J., Fortin, N., Bocher, E., Petit, G., Aumond, P., \& Guillaume, G. (2019). An open-science crowdsourcing approach for producing community noise maps using smartphones. Building and Environment, 148, 20-33. https://doi.org/10.1016/j.buildenv.2018.10.049

Rana, R., Chou, C. T., Bulusu, N., Kanhere, S., \& Hu, W. (2015). Ear-Phone: A context-aware noise mapping using smart phones. Pervasive and Mobile Computing, 17, 1-22. https://doi.org/10.1016/j.pmcj.2014.02.001

Serpanos, Y. C., Renne, B., Schoepflin, J. R., \& Davis, D. (2018). The accuracy of smartphone sound level meter applications with and without calibration. American Journal of Speech-Language Pathology, 27(4), 1319-1328. https://doi.org/10.1044/ 2018_AJSLP-17-0171
Trombetta Zannin, P. H., Coelho Ferreira, A. M., \& Szeremetta, B. (2006). Evaluation of noise pollution in urban parks. Environmental Monitoring and Assessment, 118(1-3), 423-433. https://doi.org/10.1007/s10661-006-1506-6

Wessels, P. W., \& Basten, T. G. H. (2016). Design aspects of acoustic sensor networks for environmental noise monitoring». Applied Acoustics, 110, 227-234. https://doi.org/10.1016/j.apacoust.2016.03.029

Zamora, W., Calafate, C. T., Cano, J. C., \& Manzoni, P. (2017). Accurate ambient noise assessment using smartphones. Sensors, 17(4), 917. https://doi.org/ 10.3390/s17040917

Zamorano González, B., Velázquez Narváez, Y., Peña Cárdenas, F., Ruiz Ramos, L., Monreal Aranda, O., Parra Sierra, V. \& Vargas Martínez, J. I. (2019). Exposición al ruido por tráfico vehicular y su impacto sobre la calidad del sueño y el rendimiento en habitantes de zonas urbanas». Estudios demográficos y urbanos, 34(3), 601-629. https://doi.org/10.24201/ edu.v34i3.1743

Zuo, J., Xia, H., Liu, S., \& Qiao, Y. (2016). Mapping Urban Environmental Noise Using Smartphones. Sensors (Basel, Switzerland), 16(10), 1692. https://doi.org/10.3390/s16101692 